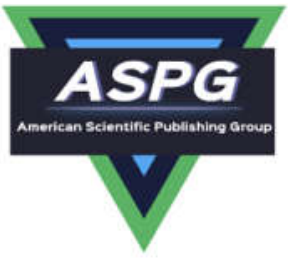

\title{
A Short Note On The Solution Of n-Refined Neutrosophic Linear Diophantine Equations
}

\author{
Rozina Ali \\ Independent researcher, Cairo, Egypt, e-mail:rozyyy123n@gmail.com
}

\begin{abstract}
This paper is dedicated to study the n-refined neutrosophic linear Diophantine equations. It provides for the first time an easy algorithm to solve these equations, with many related examples.
\end{abstract}

Keywords: n-refined neutrosophic integer, n-refined neutrosophic linear Diophantine equation.

\section{Introduction}

After the arrival of neutrosophic logic in 1995 [1], many applications in pure mathematics have been introduced. We find a huge effect of indeterminacies in topology $[2,63,66]$, matrix theory $[49,53,62]$, spaces theory $[7,8,31]$, and module theory $[6,43,51]$.

On the other hand, the inserting of indeterminacy into the ring of integers began in [11], where the neutrosophic rings were a good generalization of classical rings. See $[19,25,65]$.

Neutrosophic number theory began in [69], where the properties of neutrosophic integers have been discussed. In addition, we find deeper study in [57] for congruencies, divisibility, and Euler's theorems.

In [4], authors defined for the first time the linear Diophantine equations in neutrosophic rings, and nrefined neutrosophic rings respectively. Also, they provided an algorithm to solve these equations.

Smarandache et.al. have presented the concept of n-refined neutrosophic rings $[15,39,50]$, these rings are considered as new generalization of refined neutrosophic rings.

The concept of n-refined neutrosophic rings was used to define n-refined neutrosophic spaces and modules [5,46], n-refined neutrosophic matrices [54], and equations [52].

Through this paper, we extend the previous efforts in the study of neutrosophic linear Diophantine equations to the case of n-refined neutrosophic integers. We present an easy algorithm to solve this kind of equations by turning them into a classical corresponding system of linear Diophantine equations. Also, we illustrate some examples to clarify the validity of this algorithm. 


\section{Preliminaries}

\section{Definition 2.1: [15]}

Let $(\mathrm{R},+, \times)$ be a ring and $I_{k} ; 1 \leq k \leq n$ be $\mathrm{n}$ indeterminacies. We define $R_{n}(\mathrm{I})=\left\{a_{0}+a_{1} I+\cdots+\right.$ $\left.a_{n} I_{n} ; a_{i} \in R\right\}$ to be $\mathrm{n}$-refined neutrosophic ring. If $\mathrm{n}=2$ we get a ring which is isomorphic to 2 -refined neutrosophic ring $R\left(I_{1}, I_{2}\right)$.

Addition and multiplication on $R_{n}(\mathrm{I})$ are defined as:

$\sum_{i=0}^{n} x_{i} I_{i}+\sum_{i=0}^{n} y_{i} I_{i}=\sum_{i=0}^{n}\left(x_{i}+y_{i}\right) I_{i}, \sum_{i=0}^{n} x_{i} I_{i} \times \sum_{i=0}^{n} y_{i} I_{i}=\sum_{i, j=0}^{n}\left(x_{i} \times y_{j}\right) I_{i} I_{j}$.

Where $\times$ is the multiplication defined on the ring R.

It is easy to see that $R_{n}(\mathrm{I})$ is a ring in the classical concept and contains a proper ring $\mathrm{R}$.

\section{Definition 2.2: [15]}

Let $R_{n}(\mathrm{I})$ be an n-refined neutrosophic ring, it is said to be commutative if $x y=y x$ for each $\mathrm{x}, \mathrm{y} \in R_{n}(\mathrm{I})$, if there is $1 \in R_{n}(\mathrm{I}) \operatorname{such} 1 . x=x .1=x$, then it is called an n-refined neutrosophic ring with unity.

\section{Definition 2.3: [54]}

Let $X=A_{0}+A_{1} I_{1}+\cdots+A_{n} I_{n}$ be an n-refined neutrosophic element, we define its canonical sequence as follows:

$M_{0}=A_{0}, M_{j}=A_{0}+A_{j}+A_{j+1}+. .+A_{n} ; 1 \leq j \leq n$. For example $M_{3}=A_{0}+A_{3}+A_{4}+\cdots+A_{n}$.

\section{Remark 2.4: [54]}

The multiplication operation between two n-refined neutrosophic elements can be represented by the following equation:

$\left(A_{0}+A_{1} I_{1}+\cdots+A_{n} I_{n}\right)\left(B_{0}+B_{1} I_{1}+\cdots+B_{n} I_{n}\right)=M_{0} N_{0}+\left(M_{n} N_{n}-M_{0} N_{0}\right) I_{n}+\sum_{i=1}^{n-1}\left(M_{i} N_{i}-\right.$ $\left.M_{i+1} N_{i+1}\right) I_{i}$, where $M_{i}, N_{i}$ are the canonical sequences of $A_{0}+A_{1} I_{1}+\cdots+A_{n} I_{n}, B_{0}+B_{1} I_{1}+\cdots+$ $B_{n} I_{n}$ respectively.

\section{Definition 2.5: [54]}

Let $F_{n}(I)$ be any n-refined neutrosophic field. The n-refined linear neutrosophic equation with one variable over $F_{n}(I)$ is defined as follows:

$A X+B=0 ; A, B, X \in F_{n}(I)$. Where $A=a_{0}+a_{1} I_{1}+\cdots+a_{n} I_{n}, \mathrm{~B}=b_{0}+b_{1} I_{1}+\cdots+b_{n} I_{n}, X=x_{0}+$ $x_{1} I_{1}+\cdots+x_{n} I_{n}$.

\section{Theorem 2.6: [54]}

Let $F_{n}(I)$ be any n-refined neutrosophic field, $(*) A X+B=0$ be any n-refined linear neutrosophic equation over $F_{n}(I)$. Then $(*)$ is solvable over $F_{n}(I)$ if and only if the following classical system 
(1-) $a_{0} x_{0}+b_{0}=0$.

(2-) $\left(a_{0}+a_{n}\right)\left(x_{0}+x_{n}\right)+\left(b_{0}+b_{n}\right)=0$.

(3-) $\left(a_{0}+a_{n}+a_{n-1}\right)\left(x_{0}+x_{n}+x_{n-1}\right)+\left(b_{0}+b_{n}+b_{n-1}\right)=0$.

$(\mathrm{n}+1-)\left(a_{0}+a_{1}+\cdots+a_{n}\right)\left(x_{0}+x_{1}+\cdots+x_{n}\right)+\left(b_{0}+b_{1}+\cdots+b_{n}\right)=0$.

is solvable over the classical field $\mathrm{F}$.

\section{Main discussion}

Our work depends on the algorithm in Theorem 2.6. We generalize it to help with solving Diophantine equations.

\section{Definition 3.1:}

Let $Z_{n}(I)=\left\{t_{0}+t_{1} I_{1}+\cdots+t_{n} I_{n} ; t_{i} \in Z\right\}$ be the n-refined neutrosophic ring of integers. The following equation

$A X+B=C ; A, B, X, C \in Z_{n}(I)$. Where $A=a_{0}+a_{1} I_{1}+\cdots+a_{n} I_{n}, \mathrm{~B}=b_{0}+b_{1} I_{1}+\cdots+b_{n} I_{n}, X=x_{0}+$ $x_{1} I_{1}+\cdots+x_{n} I_{n}, C=c_{0}+c_{1} I_{1}+\cdots+c_{n} I_{n}$

is called an n-refined neutrosophic linear Diophantine equation.

\section{Example 3.2:}

Let $\mathrm{n}=3$, the following equation is an 3-refined neutrosophic linear Diophantine equation

$\left(1-I_{1}-I_{2}\right) X+\left(2+3 I_{2}-4 I_{3}\right)=I_{2}+2 I_{3}$.

\section{Theorem 3.3:}

Let $A X+B=C(*) ; A, B, X, C \in Z_{n}(I)$ be an n-refined neutrosophic linear Diophantine equation. It is solvable if and only if the following system of classical linear Diophantine equations is solvable.

(1-) $a_{0} x_{0}+b_{0}=c_{0}$.

(2-) $\left(a_{0}+a_{n}\right)\left(x_{0}+x_{n}\right)+\left(b_{0}+b_{n}\right)=c_{0}+c_{n}$.

(3-) $\left(a_{0}+a_{n}+a_{n-1}\right)\left(x_{0}+x_{n}+x_{n-1}\right)+\left(b_{0}+b_{n}+b_{n-1}\right)=c_{0}+c_{n}+c_{n-1}$.

$(\mathrm{n}+1-)\left(a_{0}+a_{1}+\cdots+a_{n}\right)\left(x_{0}+x_{1}+\cdots+x_{n}\right)+\left(b_{0}+b_{1}+\cdots+b_{n}\right)=c_{0}+c_{1}+\cdots+c_{n}$. 


\section{Proof:}

By using the canonical forms of multiplication from Definition 2.3, and Theorem 2.6, we get that the previous system is equivalent to the equation $(*)$.

\section{Theorem 3.4:}

The sufficient and necessary condition of the solvability of Diophantine equation $\left({ }^{*}\right)$ is:

$\operatorname{gcd}\left(a_{0}, b_{0}\right)\left|c_{0}, \operatorname{gcd}\left(a_{0}+a_{n}, b_{0}+b_{n}\right)\right|\left(c_{0}+c_{n}\right), \operatorname{gcd}\left(a_{0}+a_{n}+a_{n-1}, b_{0}+b_{n}+b_{n-1}\right) \mid\left(c_{0}+c_{n}+\right.$ $\left.c_{n-1}\right), \ldots \operatorname{gcd}\left(a_{0}+a_{1}+\cdots+a_{n}, b_{0}+b_{1}+\cdots+b_{n}\right), \mid\left(c_{0}+c_{1}+\cdots+c_{n}\right)$.

Proof:

The equation $(*)$ is solvable if and only if its equivalent system is solvable, according to Theorem 3.3.

By the condition of the solvability of any classical linear Diophantine equation, we get

$\operatorname{gcd}\left(a_{0}, b_{0}\right) \mid c_{0}\left(\right.$ from (1)), $\operatorname{gcd}\left(a_{0}+a_{n}, b_{0}+b_{n}\right) \mid\left(c_{0}+c_{n}\right)$

(from (2)),...

By continuing on the same way, we get the proof.

\section{Example 3.5:}

Consider the following 3-refined neutrosophic linear Diophantine equation:

$\left(1-I_{2}+I_{3}\right) X+\left(I_{2}+2 I_{3}\right)=2-I_{1}+4 I_{3}$.

We have: $A=1-I_{2}+I_{3}, B=I_{2}+2 I_{3}, C=I_{1}+4 I_{3}$, i. e. $a_{0}=1, a_{1}=0, a_{2}=-1, a_{3}=1$.

$b_{0}=0, b_{1}=0, b_{2}=1, b_{3}=2, c_{0}=0, c_{1}=1, c_{2}=0, c_{3}=4$.

We have $: \operatorname{gcd}\left(a_{0}, b_{0}\right)=1\left|0, \operatorname{gcd}\left(a_{0}+a_{3}, b_{0}+b_{3}\right)=\operatorname{gcd}(2,2)=2\right| 4, \operatorname{gcd}\left(a_{0}+a_{3}+a_{2}, b_{0}+b_{3}+\right.$ $\left.b_{2}\right)=\operatorname{gcd}(1,3)=1\left|4, \operatorname{gcd}\left(a_{0}+a_{1}+a_{2}+a_{3}, b_{0}+b_{1}+b_{2}+b_{3}\right)=\operatorname{gcd}(1,3)=1\right| 5$. This implies that the previous linear Diophantine equation is solvable.

Now, we find the solution.

The equivalent system is:

$a_{0} x_{0}+b_{0}=c_{0}$, thus $x_{0}=0 .(1)$

$\left(a_{0}+a_{3}\right)\left(x_{0}+x_{3}\right)+\left(b_{0}+b_{3}\right)=c_{0}+c_{3}$, thus $2\left(x_{0}+x_{3}\right)+2=4 .(2)$

$\left(a_{0}+a_{3}+a_{2}\right)\left(x_{0}+x_{3}+x_{2}\right)+\left(b_{0}+b_{3}+b_{2}\right)=c_{0}+c_{3}+c_{2}$, thus $\left(x_{0}+x_{3}+x_{2}\right)+3=4$.

$\left(a_{0}+a_{3}+a_{1}+a_{2}\right)\left(x_{0}+x_{3}+x_{1}+x_{2}\right)+\left(b_{0}+b_{3}+b_{1}+b_{2}\right)=c_{0}+c_{3}+c_{1}+c_{2}$, thus $\left(x_{0}+x_{1}+\right.$ $\left.x_{3}+x_{2}\right)+3=5$. (4) 
The equation (1) has a solution $x_{0}=0$. The equation (2) has a solution $x_{0}+x_{3}=1$, hence $x_{3}=1$.

The equation (2), has a solution $x_{0}+x_{3}+x_{2}=1$, hence $x_{2}=0$. The equation (4) has a solution $x_{0}+$ $x_{1}+x_{3}+x_{2}=2$, hence $x_{1}=1$.

The previous discussion means that the solution of the first n-refined neutrosophic linear Diophantine equation is $X=I_{1}+I_{3}$.

\section{Conclusion}

In this paper, we have studied for the first time the concept of n-refined neutrosophic linear Diophantine equations. We have extended the algorithm of solving a linear n-refined neutrosophic equation to be useful in the solution of n-refined neutrosophic linear Diophantine equation. Also, we have illustrated a good example to show the validity of our algorithm.

Funding:: This research received no external funding

Conflicts of Interest: The authors declare no conflict of interest

\section{References}

[1] Smarandache, F., " A Unifying Field in Logics: Neutrosophic Logic, Neutrosophy, Neutrosophic Set, Neutrosophic Probability", American Research Press. Rehoboth, 2003.

[2] Alhamido, R., and Gharibah, T., "Neutrosophic Crisp Tri-Topological Spaces", Journal of New Theory, Vol. 23 , pp.13-21. 2018.

[3] Edalatpanah. S.A., "Systems of Neutrosophic Linear Equations", Neutrosophic Sets and Systems, Vol. 33, pp. 92-104. 2020.

[4] Sankari, H., and Abobala, M., "Neutrosophic Linear Diophantine Equations With two Variables", Neutrosophic Sets and Systems, Vol. 38, pp. 22-30, 2020.

[5] Sankari, H., and Abobala, M." n-Refined Neutrosophic Modules", Neutrosophic Sets and Systems, Vol. 36, pp. 1-11. 2020.

[6] Alhamido, R., and Abobala, M., "AH-Substructures in Neutrosophic Modules", International Journal of Neutrosophic Science, Vol. 7, pp. 79-86 . 2020.

[7] Abobala, M., "AH-Subspaces in Neutrosophic Vector Spaces", International Journal of Neutrosophic Science, Vol. 6, pp. 80-86. 2020.

[8] Abobala, M.,. "A Study of AH-Substructures in $n$-Refined Neutrosophic Vector Spaces", International Journal of Neutrosophic Science", Vol. 9, pp.74-85. 2020.

[9] Hatip, A., Alhamido, R., and Abobala, M., "A Contribution to Neutrosophic Groups", International Journal of Neutrosophic Science", Vol. 0, pp. 67-76 . 2019. 
[10] Abobala, M., " $n$-Refined Neutrosophic Groups I", International Journal of Neutrosophic Science, Vol. 0, pp. 27-34. 2020.

[11] Kandasamy, V.W.B., and Smarandache, F., "Some Neutrosophic Algebraic Structures and Neutrosophic N-Algebraic Structures", Hexis, Phonex, Arizona, 2006.

[12] Agboola, A.A.A., Akinola, A.D., and Oyebola, O.Y., " Neutrosophic Rings I" , International J.Mathcombin, Vol 4,pp 1-14. 2011

[13] Agboola, A.A.A., "On Refined Neutrosophic Algebraic Structures," Neutrosophic Sets and Systems,Vol.10, pp. 99-101. 2015.

[14] Abobala, M., "Classical Homomorphisms Between Refined Neutrosophic Rings and Neutrosophic Rings", International Journal of Neutrosophic Science, Vol. 5, pp. 72-75. 2020.

[15] Smarandache, F., and Abobala, M., n-Refined neutrosophic Rings, International Journal of Neutrosophic Science, Vol. 5, pp. 83-90, 2020.

[16] Kandasamy, I., Kandasamy, V., and Smarandache, F., "Algebraic structure of Neutrosophic Duplets in Neutrosophic Rings", Neutrosophic Sets and Systems, Vol. 18, pp. 85-95. 2018.

[17] Yingcang, Ma., Xiaohong Zhang ., Smarandache, F., and Juanjuan, Z., "The Structure of Idempotents in Neutrosophic Rings and Neutrosophic Quadruple Rings", Symmetry Journal (MDPI), Vol. 11. 2019.

[18] Kandasamy, V. W. B,. Ilanthenral, K., and Smarandache, F., "Semi-Idempotents in Neutrosophic Rings", Mathematics Journal (MDPI), Vol. 7. 2019.

[19] Abobala, M., "On Some Special Substructures of Neutrosophic Rings and Their Properties, International Journal of Neutrosophic Science", Vol. 4, pp. 72-81, 2020.

[20] Smarandache, F., " An Introduction To neutrosophic Genetics", International Journal of neutrosophic Science, Vol.13, 2021.

[21] Martin, N, Smarandache, F, and Broumi, S., " Covid 19 Decision Making using Extended Plithogenic hypersoft Sets With Dual Dominent Attributes", International Journal of neutrosophic Science, Vol. 13, 2021.

[22]Agboola, A.A., "Introduction To Neutro groups", International Journal of neutrosophic Science, Vol. $6,2020$.

[23] Abobala, M., "On Some Special Substructures of Refined Neutrosophic Rings", International Journal of Neutrosophic Science, Vol. 5, pp. 59-66. 2020.

[24] Smarandache, F., and Ali, M., "Neutrosophic Triplet Group", Neural. Compute. Appl. 2019.

[25] Sankari, H., and Abobala, M.," AH-Homomorphisms In neutrosophic Rings and Refined Neutrosophic Rings", Neutrosophic Sets and Systems, Vol. 38, pp. 101-112, 2020. 
[26] Smarandache, F., and Kandasamy, V.W.B., " Finite Neutrosophic Complex Numbers", Source: arXiv. 2011.

[27]. Suresh, R., and S. Palaniammal,. "Neutrosophic Weakly Generalized open and Closed Sets", Neutrosophic Sets and Systems, Vol. 33, pp. 67-77,. 2020.

[28] Sahin, M., Olgun, N., Uluçay, V., Kargın, A., and Smarandache, F. , "A New Similarity Measure Based on Falsity Value between Single Valued Neutrosophic Sets Based on the Centroid Points of Transformed Single Valued Neutrosophic Numbers with Applications to Pattern Recognition", Neutrosophic Sets and Systems, vol. 15, pp. 31-48. 2017.

[29] Ibrahim, M.A., Agboola, A.A.A, Badmus, B.S. and Akinleye, S.A., "On refined Neutrosophic Vector Spaces I", International Journal of Neutrosophic Science, Vol. 7, pp. 97-109. 2020.

[30] Ibrahim, M.A., Agboola, A.A.A, Badmus, B.S., and Akinleye, S.A., "On refined Neutrosophic Vector Spaces II", International Journal of Neutrosophic Science, Vol. 9, pp. 22-36. 2020.

[31] Abobala, M, "n-Cyclic Refined Neutrosophic Algebraic Systems Of Sub-Indeterminacies, An Application To Rings and Modules", International Journal of Neutrosophic Science, Vol. 12, pp. 81-95 . 2020 .

[32] Smarandache, F., "Neutrosophic Set a Generalization of the Intuitionistic Fuzzy Sets", Inter. J. Pure Appl. Math., pp. 287-297. 2005.

[33] M. Ali, F. Smarandache, M. Shabir and L. Vladareanu., "Generalization of Neutrosophic Rings and Neutrosophic Fields", Neutrosophic Sets and Systems, vol. 5, pp. 9-14, 2014.

[34] Anuradha V. S., "Neutrosophic Fuzzy Hierarchical Clustering for Dengue Analysis in Sri Lanka", Neutrosophic Sets and Systems, vol. 31, pp. 179-199. 2020.

[35] Olgun, N., and Hatip, A., "The Effect Of The Neutrosophic Logic On The Decision Making, in Quadruple Neutrosophic Theory And Applications", Belgium, EU, Pons Editions Brussels,pp. 238-253. 2020 .

[36] Zadeh, L. "Fuzzy Sets", Inform and Control, 8, pp.338-353. 1965.

[37] Turksen, I., "Interval valued fuzzy sets based on normal forms", Fuzzy Sets and Systems, 20, pp.191-210, 1986. 1986.

[38] Chalapathi, T., and Madhavi, L., "Neutrosophic Boolean Rings", Neutrosophic Sets and Systems, Vol. 33, pp. 57-66. 2020.

[39] Abobala, M., "Classical Homomorphisms Between n-refined Neutrosophic Rings", International Journal of Neutrosophic Science", Vol. 7, pp. 74-78. 2020.

[40] Agboola, A.A.A,. Akwu, A.D,. and Oyebo, Y.T., " Neutrosophic Groups and Subgroups", International .J .Math. Combin, Vol. 3, pp. 1-9. 2012. 
[41] Smarandache, F., " $n$-Valued Refined Neutrosophic Logic and Its Applications in Physics", Progress in Physics, 143-146, Vol. 4, 2013.

[42] Adeleke, E.O., Agboola, A.A.A.,and Smarandache, F., "Refined Neutrosophic Rings I", International Journal of Neutrosophic Science, Vol. 2(2), pp. 77-81. 2020.

[43] Hatip, A., and Abobala, M., "AH-Substructures In Strong Refined Neutrosophic Modules", International Journal of Neutrosophic Science, Vol. 9, pp. 110-116 . 2020.

[44] Hatip, A., and Olgun, N., "On Refined Neutrosophic R-Module", International Journal of Neutrosophic Science, Vol. 7, pp.87-96. 2020.

[45] Chakraborty, A., Banik, B., Mondal, S.P., and Alam, S., "Arithmetic and Geometric Operators of Pentagonal Neutrosophic Number and its Application in Mobile Communication Service Based MCGDM Problem", Neutrosophic Sets and Systems, vol. 32, pp. 61-79. 2020.

[46] Smarandache F., and Abobala, M., "n-Refined Neutrosophic Vector Spaces", International Journal of Neutrosophic Science, Vol. 7, pp. 47-54. 2020.

[47] Sankari, H., and Abobala, M., "Solving Three Conjectures About Neutrosophic Quadruple Vector Spaces", Neutrosophic Sets and Systems, Vol. 38, pp. 70-77. 2020.

[48] Adeleke, E.O., Agboola, A.A.A., and Smarandache, F., "Refined Neutrosophic Rings II", International Journal of Neutrosophic Science, Vol. 2(2), pp. 89-94. 2020.

[49] Abobala, M., "On Refined Neutrosophic Matrices and Their Applications In Refined Neutrosophic Algebraic Equations", Journal Of Mathematics, Hindawi, 2021

[50] Abobala, M., "A Study of Maximal and Minimal Ideals of n-Refined Neutrosophic Rings", Journal of Fuzzy Extension and Applications, Vol. 2, pp. 16-22, 2021.

[51] Abobala, M., " Semi Homomorphisms and Algebraic Relations Between Strong Refined Neutrosophic Modules and Strong Neutrosophic Modules", Neutrosophic Sets and Systems, Vol. 39, 2021.

[52] Abobala, M., "On Some Neutrosophic Algebraic Equations", Journal of New Theory, Vol. 33, 2020.

[53] Abobala, M., "On The Representation of Neutrosophic Matrices by Neutrosophic Linear Transformations", Journal of Mathematics, Hindawi, 2021.

[54] Abobala, M., "On Some Algebraic Properties of n-Refined Neutrosophic Elements and n-Refined Neutrosophic Linear Equations", Mathematical Problems in Engineering, Hindawi, 2021

[55] Kandasamy V, Smarandache F., and Kandasamy I., Special Fuzzy Matrices for Social Scientists . Printed in the United States of America,2007, book, 99 pages.

[56] Khaled, H., and Younus, A., and Mohammad, A., " The Rectangle Neutrosophic Fuzzy Matrices", Faculty of Education Journal Vol. 15, 2019. (Arabic version). 
[57] Abobala, M., "Partial Foundation of Neutrosophic Number Theory", Neutrosophic Sets and Systems, Vol. $39,2021$.

[58] F. Smarandache, Neutrosophic Theory and Applications, Le Quy Don Technical University, Faculty of Information technology, Hanoi, Vietnam, $17^{\text {th }}$ May 2016.

[59] Sankari, H, and Abobala, M., " On A New Criterion For The Solvability of non Simple Finite Groups and m-Abelian Solvability, Journal of Mathematics, Hindawi, 2021.

[60] Giorgio, N, Mehmood, A., and Broumi, S.," Single Valued neutrosophic Filter", International Journal of Neutrosophic Science, Vol. 6, 2020.

[61] Es, Haydar, A., "A Note On neutrosophic Soft Menger Topological Spaces", International Journal of Neutrosophic Science, Vol.7, 2020.

[62] Abobala, M., Hatip, A., Olgun, N., Broumi, S., Salama, A,A., and Khaled, E, H., The algebraic creativity In The Neutrosophic Square Matrices, Neutrosophic Sets and Systems, Vol. 40, pp. 1-11, 2021.

[63]Alhamido, K., R., "A New Approach of neutrosophic Topological Spaces", International Journal of neutrosophic Science, Vol.7, 2020.

[64] Chellamani, P., and Ajay, D., "Pythagorean neutrosophic Fuzzy Graphs", International Journal of Neutrosophic Science, Vol. 11, 2021.

[65] Abobala, M., "On Some Special Elements In Neutrosophic Rings and Refined Neutrosophic Rings", Journal of New Theory, vol. 33, 2020.

[66]Milles, S, Barakat, M, and Latrech, A., " Completeness and Compactness In Standard Single Valued neutrosophic Metric Spaces", International Journal of Neutrosophic Science, Vol.12 , 2021.

[67] Agboola, A.A., "Introduction to Anti Groups", International Journal of Neutrosophic Science, vol.12, 2021.

[68] Sankari, H, and Abobala, M, " A Contribution to m-Power Closed Groups", UMM-Alqura University Journal for Applied Sciences, KSA, 2020.

[69] Ceven, Y., and Tekin, S., " Some Properties of Neutrosophic Integers", Kırklareli University Journal of Engineering and Science, Vol. 6, pp.50-59, 2020. 